\title{
Can Light-Shaped Visual Prompt Promote Individuals' Lights off Behaviors More Effectively than Ordinary One?
}

\author{
Zhuli Feng, Fuzhen Zhang* \\ Department of Marxism, China Pharmaceutical University, Nanjing, China \\ Email: *18851725530@163.com
}

How to cite this paper: Feng, Z. L., \& Zhang, F. Z. (2019). Can Light-Shaped Visual Prompt Promote Individuals' Lights off Behaviors More Effectively than Ordinary One? Psychology, 10, 79-87. https://doi.org/10.4236/psych.2019.101006

Received: December 10, 2018

Accepted: January 14, 2019

Published: January 17, 2019

Copyright (๑) 2019 by author(s) and Scientific Research Publishing Inc. This work is licensed under the Creative Commons Attribution International License (CC BY 4.0).

http://creativecommons.org/licenses/by/4.0/

\section{(c) (i) Open Access}

\begin{abstract}
Lights off behavior makes contribution to energy saving, meeting the realistic requirements of building a sustainable society. Several studies investigated the effectiveness of prompt on energy saving behavior, but few focused on how to make prompt more effective by "packaging". In this current study, light-shaped prompt was designed with inspiration from light itself and 18 washrooms were studied in an ABAB-B method to verify whether the light-shaped visual prompt could promote lights off behaviors more than ordinary one, with a duration of 9 weeks, during which a one-week follow-up was conducted 4 weeks after $A B A B$ was finished, to investigate the sustained effect of light-shaped visual prompt on lights off behaviors. Study showed that light-shaped visual prompt could exert more effective influence on lights off behaviors than the ordinary one. Individuals' lights off behavior persisted throughout the follow-up period. This study provided a new perspective for improving the effectiveness of prompt. But further study on more well-designed visual prompt of diverse shape was necessary for the maximization of effectiveness and expanded application.
\end{abstract}

\section{Keywords}

Light-Shaped Visual Prompt, Ordinary Visual Prompt, Lights off Behaviors, Promote

\section{Introduction}

Energy is vital to human survival and reproduction. Except industrial and commercial sectors, individual energy saving behavior is of great importance for reducing the environmental load and building a sustainable society. Facing the fact 
that young people perform less internalized motivation to act pro-environmentally than their parents (Alice \& John, 2017), and lower practice rates are particularly common among younger generations (Lee, Kurisu, \& Hanaki, 2013; Kurisu \& Bortoleto, 2011). It is really both urgent and meaningful to promote energy saving behaviors of individuals. Electricity was an important energy resource and the solid, lignite used in electricity generation particularly was assessed as an energy source with the most negative impact on the emissions of greenhouse gas (Simona et al., 2018). Washroom was a public place where electricity was easily to be wasted because of the tragedy of commons, which resulted in the emissions of more greenhouse gas. The key to improving this negative phenomenon is to promote lights off behaviors of individuals.

Visual prompt is a method of using indicative or prohibitive information on visual carrier to exert behavioral intervention, so as to persuade individuals to perform as expected. For decades, with the advantage of simplicity of production and low price, visual prompt has been studied by lots of researchers in promoting pro-environmental behaviors (PEBs) of individuals, such as behavioral intervention for household food waste recycling, energy saving and compost-supportive behaviors (Shearer et al., 2017; Pandey et al., 2016; Tetlow et al., 2014; Sussman et al., 2013); significant and positive results inspired us that visual prompt was an effective strategy for promoting lights off behaviors. At present, studies on encouraging lights off behaviors by visual prompt mostly focused on the evaluation of effectiveness, few studies work on how to make visual prompt more effective by packaging, such as making the shape of prompt more diverse, adjusting the size of visual prompt and so on (Sussman \& Gifford, 2012). Therefore, study on visual prompt in promoting individual lights off behaviors was rarely and insufficient. Based on above issues, this current study attempted to verify whether light-shaped visual prompt could promote individuals' lights off behaviors more than ordinary one, expecting to provide a new perspective for improving the effectiveness of visual prompt.

\section{Method}

\subsection{Washrooms and Volunteers}

Eighteen washrooms (all in normal use) of five teaching buildings were studied in a medium-sized school with about 16,144 students in China. The number of washrooms for males was equal to the ones for females. Eighteen volunteers were needed to observe and record the state of the light during the study, but we chose 20 of them (mainly undergraduate students), expecting another two more as a supplement to other volunteers to prevent unexpected situation. Volunteers were required to check and record the state of washroom light twice a day with at least a two-hours interval between observations. And it was usually $1 \mathrm{~h}$ for each observation. Each volunteer checked the same washroom from the beginning to the end. The state of washroom light was set by "on, off, on but in use". If the washroom was occupied, the observers were excepted to come later or wait 
outside the washroom till it was empty to complete their observation. Volunteers were forbidden to change the state of washroom light, for example, if the washroom light was on, then leave it on, but not turn it off for any energy-saving purpose.

\subsection{Procedure and Observation}

This experiment lasted for 9 weeks with an interval of 4 weeks. The former 4 weeks were spent to observe the effectiveness of light-shaped visual prompt in promoting lights off behavior compared with ordinary one in two consecutive cycles. And then a one-week follow-up was conducted 4 weeks (no observation were recorded) later to verify if light-shaped visual prompt could exert sustained influence on individuals' lights off behaviors. Observation at weekends took place as usual with weekdays during experimental weeks (5 in total).

ABAB-B method was adopted in this study, which refers to two continuous cycles (ABAB) and a follow-up (B). Phase A was set as baseline and phase $B$ was conducted under target conditions to investigate the difference between $A$ and $B$. Considering that visual prompt has been proven by studies to promote energy saving behaviors effectively, baseline phase A 1 in this experiment was set with ordinary visual prompt, but not without visual prompt in all washrooms, aiming to focus on the comparison of the effectiveness of two kinds visual prompt. In phase A1, ordinary visual prompt was pasted to the wall near the light switch (if there were more than one light switches in the washroom, choose the one near the entrance), and observers were required to check and record the state of washroom light (on, off, on but in use) for a week. During course B1, in order to investigate if visual prompt of diverse shape could promote lights off behaviors more effectively, 9 washrooms (all male washrooms) were pasted with light-shaped visual prompt and another 9 ones (all female washrooms) still kept the initial ones. In the second baseline phase (A2), light-shaped visual prompt were removed and replaced by ordinary ones, and observation lasted for a week, including weekends. During the second intervention phase (B2), which lasted for a week, all ordinary visual prompt were replaced by light-shaped ones. All visual prompt were then left on the wall of all washrooms without any change after B2 was finished. 4 weeks later, one-week follow-up (B3) was conducted to investigate the sustained effectiveness of light-shaped visual prompt exerting on individuals' lights off behavior.

\subsection{Details for Visual Prompt}

\subsubsection{Light-Shaped Visual Prompt}

The inspiration of light-shaped visual prompt (Figure 1) was from the light itself. The cute and colorful cartoon image could be more attractive to draw individuals' attention to pro-"lights off". Except personal thoughts, the prompt was designed as well as consultations with teachers, classmates and friends. Content used in prompt can exert large influence on energy conservation behavior (Bergquist \& Nilsson, 2016), and research showed that people exposed to more 
positive pro-environmental descriptive norms were more likely to engage themselves in pro-environmental behaviors (PEBs) (Bissing-Olson et al., 2016; Cialdini, Reno, \& Kallgren, 1990; Nolan et al., 2008), while existential threat decreases target behavior (Buttlar et al., 2017). With that in mind, the content of prompt was modified in descriptive and positive norm information to maximize politeness and informativeness, reading "turning off the lights for even 5 seconds saves electricity. Please turn off the lights for your bright future". " 5 seconds", "bright future" and "turn off" were emphasized by the way of being enlarged and bold to make a clearer sight and strengthen the effectiveness. The green background was designed to appeal green action.

\subsubsection{The Ordinary Visual Prompt}

The ordinary visual prompt (Figure 2) was designed according to ones commonly seen in some washrooms of daily life, consisting of content and picture. In terms of content, two kind of visual prompt shared the same. The background

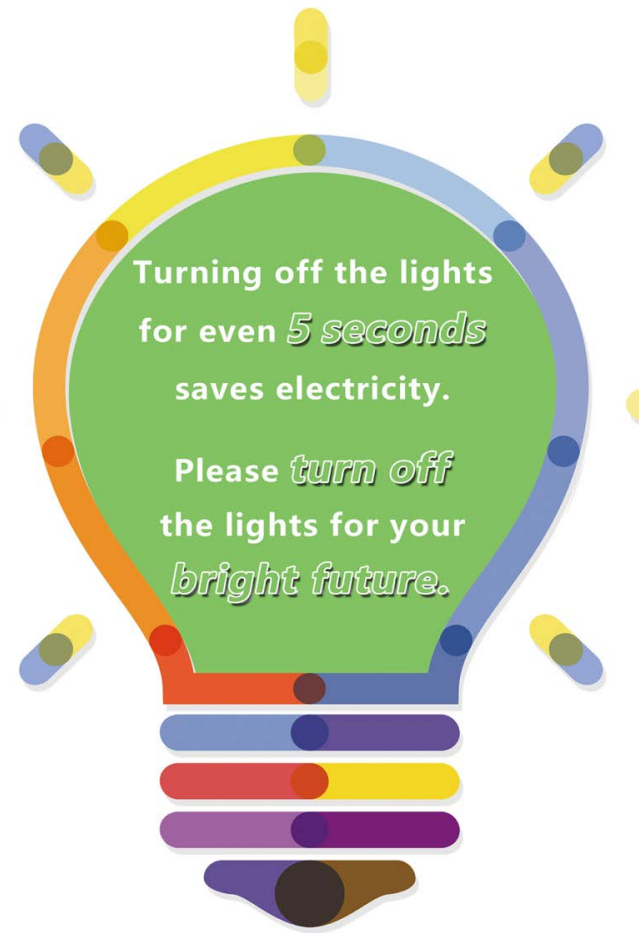

Figure 1. The light-shaped visual prompt.

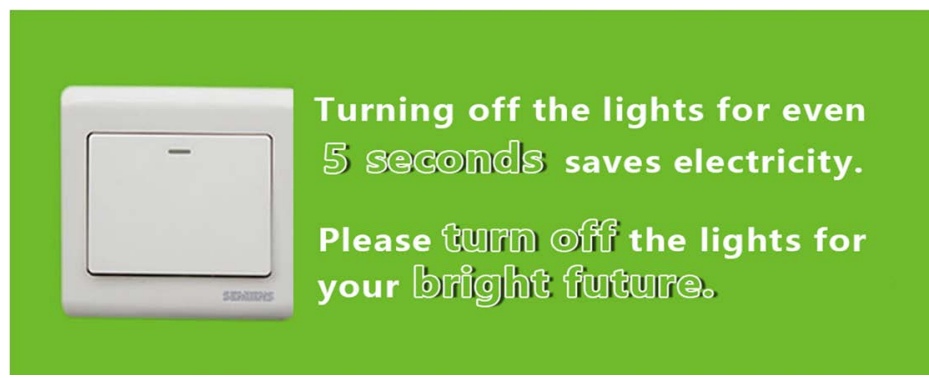

Figure 2. The ordinary visual prompt. 
light green, aiming to guide individuals to make pro-environmental decisions. In was the left of the prompt, there was a picture of light switch, which attempted to remind individuals of turning off the lights in time.

\section{Results}

\subsection{About the Prompt}

Considering that visual prompt might be removed by cleaners or students during the experiment, which could exert great influence on observation and data collection. Volunteers were asked to write some notice and the end date on the blackboard of classrooms nearby before the experiment was conducted, to tell the students not to remove the visual prompt and we would really appreciate it if they re-paste the dropped visual prompt to the original location. Similarly, the cleaners were informed that the visual prompt were only for experimental use and would be removed when finished. Luckily, no visual prompt was removed during the entire period, only one ordinary prompt dropped on floor during B1 (after $5 \mathrm{pm}$ to $9 \mathrm{am}$ the next day) and was re-pasted timely. Considering that the prompt dropped outside the observation time, observations of this washroom was included in statistical analysis. No other situations unusual happened.

\subsection{About the Observation Time}

The volunteers were asked to observe and record the state of washroom light twice a day and one hour each time, with an interval of at least two hours between observations. But the time was not uniformly defined and was determined by the observers themself. According to the record wrote by the volunteers, $74 \%$ ( $\mathrm{n}=1909)$ of the first observation was conducted from 8 am to $11 \mathrm{am}$, while the remaining $26 \%(n=671)$ was made from half past one to three in the afternoon. The second observation occurred in the afternoon from $2 \mathrm{pm}$ to $9 \mathrm{pm}$. Which was consistent with the school's course schedule on the whole.

\subsection{About the Lights off Behavior}

Lights off behavior rates $=N_{\text {off }} / N_{\text {total }} \times 100 \%$

$N_{\text {off }}$ : number of lights off behavior each day (phase/entire period).

$N_{\text {total }}$ : observations of each day (phase/entire period).

Totally, as was shown in Table 1, 2580 observations were obtained in 18 washrooms during the entire study. Thanks to advanced notice of not removing the visual prompt unauthorizedly, only one visual prompt dropped during B1, but outside the observation time. Therefore, all observations were valid to be included in data analysis. The calculation method of lights off behavior rates was as shown in the above publicity. On the whole, the lights off behavior rates was $38.94 \%(n=1005)$, the percentage of lights on was $36.41 \%(n=939)$, and the percentage of lights on but in use was $24.65 \%(n=636)$. As for each phase, $17.37 \%(\mathrm{n}=448)$ of the observations were obtained during baseline phase A1, and $17.65 \%(n=455)$ occurred in phase B1, including 58.68\% $(n=267)$ acquired 
in washrooms with light-shaped visual prompt and $41.32 \%(n=188)$ obtained in washrooms with the ordinary one. During phase A2, 17.09\% $(n=441)$ of the observations were made and $26.33 \%(n=679)$ took place in phase B2. In the follow-up (B3), 21.57\% ( $\mathrm{n}=557)$ were obtained by the observer.

The dotted line in Figure 3 was a trend line, which showed that the lights off behavior rates of each day during phase A1, B1 and B2 had a significant upward trend. Statistical analysis indicated that there was significant difference between A1and B1 ( $\mathrm{P}=0.007), \mathrm{A} 2$ and B2 $(\mathrm{P}=0.000)$, which meant that light-shaped visual prompt could exert more effective influence on lights off behaviors of individuals. B3 was conducted 4 weeks after B2 was finished to investigate whether the light-shaped visual prompt had sustained effectiveness on lights off behaviors, and data analysis indicated that there was no significance between B2 and B3 ( $\mathrm{P}=0.393)$, supporting the expectation that light-shaped prompt had sustained effect on lights off behavior of individuals.

Table 1. Observations of each phase.

\begin{tabular}{cc}
\hline Phase & Observations \\
A1 & $17.37 \%(\mathrm{n}=448)$ \\
B1 & $17.65 \%(\mathrm{n}=455)$ \\
A2 & $17.09 \%(\mathrm{n}=441)$ \\
B2 & $26.33 \%(\mathrm{n}=679)$ \\
B3 & $21.57 \%(\mathrm{n}=557)$ \\
& On: $36.41 \%(\mathrm{n}=939)$ \\
Total & Off: $38.94 \%(\mathrm{n}=1005)$
\end{tabular}

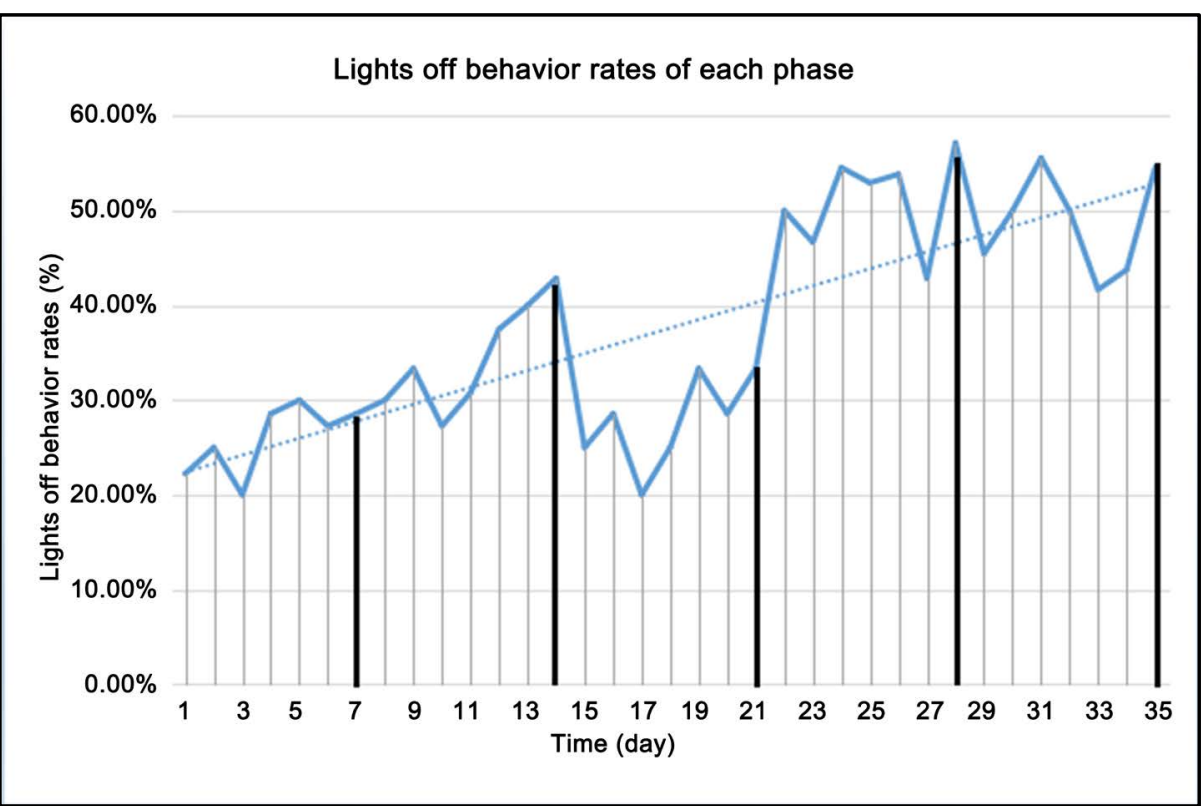

Figure 3. Lights off behavior rates of each phase. 


\section{Discussion}

Light-shaped visual prompts have more significant and sustained impact on individuals' lights off behavior than ordinary ones. The reason is that colourful and vivid shape made the prompt more attractive to meet the psychological needs of individuals and draw more people's attention and recognition of target behavior. The research inspires us that the effectiveness of prompt can be enhanced by shape packaging, pointing out a meaningful way for the future research. Limitations existed in this study. Firstly, only one shape (light) was designed and studied in the experiment, which brought about difficulties in expanding application of results to other field, such as water saving. Based on that, further study on more shapes is necessary, and considering combination of feedback, visual prompts, incentives or goals could reduce electricity use effectively (Clayton \& Nesnidol, 2017; Bekker et al., 2010), research of investigating whether the replacement of diverse-shape prompt could motivate electricity saving ulteriorly is an effective way to maximize the effectiveness of encouraging pro-environmental behavior by mixed means. Secondly, it is difficult to infer precise causality because of the lack of strict control of experimental conditions. Individuals' lights off behavior was influenced by lots of factors, such as environmental awareness and modeling, So we can't be certain that each observation was of a separate individual choice or a given person's lights off behaviour may have been observed multiple times during the entire study. Measures of controlling experimental conditions to make the study more independent are necessary, but we still haven't worked it out.

\section{Conclusion}

This research investigated the effectiveness of lights off behavioral intervention exerted by light-shaped visual prompt and ordinary ones, showing that the former had more significant and sustained effect. Actually, the precondition of effective behavioral intervention by prompt is to draw individuals' attention to indicative information. Prompt of diverse shapes is more attractive to make individuals focus and do as what the prompt reads, inspiring us that further study on more kinds of shapes is meaningful to enhance the effectiveness of prompt. Compared with other energy saving, such as gas, coal and so on, lights off behavior can only contribute to low electricity conservation. It seems that it is not necessary enough to cost so much in this field. But considering that vivid prompt can promote lights off behavior, it can also be applied in other conservation behaviors, such as "Please turn off the tap", and the shape of prompt can be designed as water drops or more shapes should be studied and chosen to maximize the effectiveness of visual prompt. In addition to all above, cost of prompt is relatively cheap, which means that it is practicable and easier to apply on a large scale.

\section{Conflicts of Interest}

The author declares no conflicts of interest regarding the publication of this paper. 


\section{References}

Alice, G., \& John, T. (2017). Why Young People Do Things for the Environment: The Role of Parenting for Adolescents' Motivation to Engage in Pro-Environmental Behaviour. Environmental Psychology, 54, 11-19.

https://doi.org/10.1016/j.jenvp.2017.09.005

Bekker, M. J., Cumming, T. D., Osborne, N. K. P., Bruining, A. M., McClean, J. I., \& Leland Jr., L. S. (2010). Encouraging Electricity Savings in a University Residential Hall through a Combination of Feedback, Visual Prompts, and Incentives. Applied Behavior Analysis, 43, 327-331. https://doi.org/10.1901/jaba.2010.43-327

Bergquist, M, \& Nilsson, A. (2016). I Saw the Sign: Promoting Energy Conservation via Normative Prompts. Environmental Psychology, 46, 23-31.

https://doi.org/10.1016/j.jenvp.2016.03.005

Bissing-Olson, M. J., Fielding, K. S., \& Iyer, A. (2016). Experiences of Pride, Not Guilt, Predict Pro-Environmental Behavior When Pro-Environmental Descriptive Norms Are More Positive. Environmental Psychology, 45, 145-153.

https://doi.org/10.1016/j.jenvp.2016.01.001

Buttlar, B., Latz, M., \& Walther, E. (2017). Breaking Bad: Existential Threat Decreases Pro-Environmental Behavior. Basic \& Applied Social Psychology, 39, 153-166. https://doi.org/10.1080/01973533.2017.1296360

Cialdini, R. B., Reno, R. R., \& Kallgren, C. A. (1990). A Focus Theory of Normative Conduct: Recycling the Concept of Norms to Reduce Littering in Public Places. Journal of Personality and Social Psychology, 58, 1015-1026. https://doi.org/10.1037/0022-3514.58.6.1015

Clayton, M., \& Nesnidol, S. (2017). Reducing Electricity Use on Campus: The Use of Prompts, Feedback, and Goal Setting to Decrease Excessive Classroom Lighting. Organizational Behavior Management, 37, 196-206. https://doi.org/10.1080/01608061.2017.1325823

Kurisu, K. H., \& Bortoleto, A. P. (2011). Comparison of Waste Prevention Behaviors among Three Japanese Megacity Regions in the Context of Local Measures and Socio-Demographics. Waste Management, 31, 1441-1449.

Lee, H., Kurisu, K., \& Hanaki, K. (2013). Influential Factors on Pro-Environmental Behaviors-A Case Study in Tokyo and Seoul. Low Carbon Economy, 4, 104-116. https://doi.org/10.4236/lce.2013.43011

Nolan, J. M., Schultz, P. W., Cialdini, R. B., Griskevicius, V., \& Goldstein, N. (2008). Normative Social Influence Is under Detected. Personality and Social Psychology Bulletin, 34, 913-923. https://doi.org/10.1177/0146167208316691

Pandey, N., Diller, J. W., \& Miller, L. S. (2016). E-Mailed Prompts and Feedback Messages to Reduce Energy Consumption: Testing Mechanisms for Behavior Change by Employees at a Green University. Organizational Behavior Management, 36, 332-345. https://doi.org/10.1080/01608061.2016.1201034

Shearer, L., Gatersleben, B., Morse, S., Smyth, M., \& Hunt, S. (2017). A Problem Unstuck? Evaluating the Effectiveness of Sticker Prompts for Encouraging Household Food Waste Recycling Behaviour. Special Thematic Issue: Urban Mining and Circular Economy, Waste Management, 60, 164-172. https://doi.org/10.1016/j.wasman.2016.09.036

Simona, J., Dorota, B.-K., Pavlína, P., Jerzy, K., \& Agata, B. (2018). Greenhouse Gas Emission Assessment from Electricity Production in the Czech Republic. Environments, $5,17$. 
https://doi.org/10.3390/environments5010017

Sussman, R., \& Gifford, R. (2012). Please Turn off the Lights: The Effectiveness of Visual Prompts. Applied Ergonomics, 43, 596-603. https://doi.org/10.1016/j.apergo.2011.09.008

Sussman, R., Greeno, M., Gifford, R., \& Scannell, L. (2013). The Effectiveness of Models and Prompts on Waste Diversion: A Field Experiment on Composting by Cafeteria Patrons. Applied Social Psychology, 43, 24-34. https://doi.org/10.1111/j.1559-1816.2012.00978.x

Tetlow, R. M., Beaman, C. P., Elmualim, A. A., \& Couling, K. (2014). Simple Prompts Reduce Inadvertent Energy Consumption from Lighting in Office Buildings. Building and Environment, 81, 234-242. https://doi.org/10.1016/j.buildenv.2014.07.003 